\title{
GENERALIZED FRACTIONAL CALCULUS TO A SUBCLASS OF ANALYTIC FUNCTIONS FOR OPERATORS ON HILBERT SPACE
}

\author{
YONG CHAN KIM, JAE HO CHOI, AND JIN SEOP LEE \\ Department of Mathematics \\ Yeungnam University \\ Gyongsan 712-749, KOREA
}

(Received May 20, 1996 and in revised form July 2, 1996)

\begin{abstract}
In this paper, we investigate some generalized results of applications of fractional integral and derivative operators to a subclass of analytic functions for operators on Hilbert space.
\end{abstract}

KEY WORDS AND PHRASES: Multivalent function, Fractional calculus, Riesz-Dunford integral. 1991 AMS SUBJECT CLASSIFICATION CODES: 30C45, 33C20.

\section{INTRODUCTION AND DEFINITIONS}

Let $\mathcal{A}$ denote the class of functions of the form:

$$
f(z)=\sum_{n=0}^{\infty} a_{n+1} z^{n+1} \quad\left(a_{1}:=1\right),
$$

which are analytic in the open unit disk

$$
\mathcal{U}=\{z: z \in \mathbb{C} \text { and }|z|<1\} .
$$

Also let $\mathcal{S}$ denote the class of all functions in $\mathcal{A}$ which are univalent in the unit disk $\mathcal{U}$.

Let $\mathcal{S}_{0}(\alpha, \beta, \gamma, p)$ denote the class of functions

$$
f(z)=z^{p}-\sum_{n=1}^{\infty} a_{n+p} z^{n+p} \quad\left(a_{n+p} \geq 0\right),
$$

which are analytic and p-valent in $\mathcal{U}$ and satisfy the condition

$$
\left|\frac{z f^{\prime}(z)}{f(z)}-p\right|<\beta\left|\alpha \frac{z f^{\prime}(z)}{f(z)}+(p-\gamma)\right|
$$

for $0 \leq \alpha \leq 1,0<\beta \leq 1,0 \leq \gamma<p, p \in \mathbb{N}$ and $z \in \mathcal{U}$. See Lee et al [1] for further information on them. It is easily found that $\mathcal{S}_{0}(\alpha, \beta, \gamma, p) \subset \mathcal{A}$ when $p=1$.

Let $a, b$, and $c$ be complex numbers with $c \neq 0,-1,-2, \cdots$. Then the Gaussian hypergeometric function ${ }_{2} F_{1}(z)$ is defined by

$$
\begin{aligned}
{ }_{2} F_{1}(z) & \equiv{ }_{2} F_{1}(a, b ; c ; z) \\
& :=\sum_{n=0}^{\infty} \frac{(a)_{n}(b)_{n}}{(c)_{n}} \frac{z^{n}}{n !},
\end{aligned}
$$

where $(\lambda)_{n}$ is the Pochhammer symbol defined, in terms of the Gamma function, by

$$
\begin{aligned}
(\lambda)_{n} & :=\frac{\Gamma(\lambda+n)}{\Gamma(\lambda)} \\
& = \begin{cases}1 & (n=0) \\
\lambda(\lambda+1) \cdots(\lambda+n-1) & (n \in \mathbb{N}:=\{1,2,3, \cdots\}) .\end{cases}
\end{aligned}
$$


Let $A$ be a bounded linear operator on a complex Hilbert space $\mathcal{H}$. For a complex valued function $f$ analytic on a domain $E$ of the complex plane containing the spectrum $\sigma(A)$ of $A$ we denote $f(A)$ as Riesz-Dunford integral [2, p. 568], that is,

$$
f(A):=\frac{1}{2 \pi i} \int_{C} f(z)(z I-A)^{-1} d z
$$

where $I$ is the identity operator on $\mathcal{H}$ and $C$ is positively oriented simple closed rectifiable contour containing $\sigma(A)$.

Also $f(A)$ can be defined by the series $f(A)=\Sigma_{n=0}^{\infty} \frac{f^{(n)}(0)}{n^{1}} A^{n}$ which converges in the norm topology [3].

Xiaopei [4] defined $\mathcal{S}_{0}(\alpha, \beta, \gamma, p ; A)$ by the class of functions

$$
f(z)=z^{p}-\sum_{n=1}^{\infty} a_{n+p} z^{n+p} \quad\left(a_{n+p} \geq 0\right),
$$

which is analytic and p-valent in $\mathcal{U}$ and satisfies the condition,

$$
\left\|A f^{\prime}(A)-p f(A)\right\|<\beta\left\|\alpha A f^{\prime}(A)+(p-\gamma) f(A)\right\|
$$

for $0 \leq \alpha \leq 1,0<\beta \leq 1,0 \leq \gamma<p, p \in \mathbb{N}$ and all operators $A$ with $\|A\|<1$ and $A \neq \theta(\theta$ denotes the zero operator on $\mathcal{H}$ ).

Let $A^{*}$ denote the conjugate operator of $A$.

DEFINITION 1 ([4]). The fractional integral for operator of order $a$ is defined by

$$
D_{A}^{-a} f(A)=\frac{1}{\Gamma(a)} \int_{0}^{1} A^{a} f(t A)(1-t)^{a-1} d t
$$

where $a>0$ and $f(z)$ is an analytic function in a simply-connected region of the z-plane containing the origin.

DEFINITION 2 ([4]). The fractional derivative for operator of order $a$ is defined by

$$
D_{A}^{a} f(A)=\frac{1}{\Gamma(1-a)} g^{\prime}(A),
$$

where $g(z)=\int_{0}^{1} z^{1-a} f(t z)(1-t)^{-a} d t(0<a<1)$ and $f(z)$ is an analytic function in a simplyconnected region of the $z$-plane containing the origin.

Srivastava et al. [5] introduced a fractional integral operator $I_{0, z}^{a, b, c}$ defined by (cf. [6])

$$
\begin{gathered}
I_{0, z}^{a, b, c} f(z)=\frac{z^{-b}}{\Gamma(a)} \int_{0}^{1}(1-t)^{a-1}{ }_{2} F_{1}(a+b,-c ; a ; 1-t) f(t z) d t \\
(a>0 ; b, c \in \mathbb{R} ; f(z) \in \mathcal{A})
\end{gathered}
$$

and Owa et al. [7] studied the fractional operator $J_{0, z}^{a, b, c}$ defined by (see also Kim et al. [8])

$$
J_{0, z}^{a, b, c} f(z)=\frac{\Gamma(2-b) \Gamma(2+a+c)}{\Gamma(2-b+c)} z^{b} I_{0, z}^{a, b, c} f(z) \quad(f \in \mathcal{A}) .
$$

The fractional derivative operator $D_{0, z}^{a, b, c}$ is defined by (cf. [9])

$$
\begin{aligned}
D_{0, z}^{a, b, c} f(z)=\frac{d}{d z} & \left(\frac{z^{-b}}{\Gamma(1-a)} \int_{0}^{1}(1-t)^{-a}{ }_{2} F_{1}(b-a+1,-c ; 1-a ; 1-t) f(t z) d t\right) \\
& (0 \leq a<1 ; b, c \in \mathbb{R} ; f(z) \in \mathcal{A}) .
\end{aligned}
$$

And we define $D_{0, z}^{n+a, b, c}$ by 


$$
D_{0, z}^{n+a, b, c} f(z)=\frac{d^{n}}{d z^{n}} D_{0, z}^{a, b, c} f(z) .
$$

For all invertible operator $A$, we introduce the following definition:

DEFINITION 3. The fractional integral operator for operator $I_{0, A}^{a, b, c}$ is defined by

$$
I_{0, A}^{a, b, c} f(A)=\frac{1}{\Gamma(a)} \int_{0}^{1} A^{-b}{ }_{2} F_{1}(a+b-c ; a ; 1-t) f(t A)(1-t)^{a-1} d t,
$$

where $a>0$ and $b, c \in \mathbb{R}$.

The fractional derivative operator for operator $D_{0, A}^{a, b, c}$ is defined by

$$
D_{0, A}^{a, b, c} f(A)=\frac{1}{\Gamma(1-a)} g^{\prime}(A)
$$

where

$$
g(z)=\int_{0}^{1} z^{-b}{ }_{2} F_{1}(b-a+1,-c ; 1-a ; 1-t) f(t z)(1-t)^{-a} d t,
$$

$0<a<1$ and $b, c \in \mathbb{R}$. In both (1.14) and (1.15) $f(z)$ is an analytic function in a simply-connected region of the $z$-plane containing the origin with the order

$$
f(z)=O\left(|z|^{\epsilon}\right), \quad z \rightarrow 0,
$$

where $\epsilon>\max \{0, b-c\}-1$ and the multiplicity of $(1-t)^{a-1}$ is in (1.14) (and that of $(1-t)^{-a}$ in (1.15)) removed by requiring $\log (1-t)$ to be real when $1-t>0$.

We note that

$$
I_{0, A}^{a,-a, c} f(A)=D_{A}^{-a} f(A) \quad \text { and } \quad D_{0, A}^{a, a-1, c} f(A)=D_{A}^{a} f(A) .
$$

The object of this paper is to prove the distortion theorems of fractional integral and derivative operators to $\mathcal{S}_{0}(\alpha, \beta, \gamma, p ; A)$.

\section{RESULTS}

LEMMA 1 (Xiaopei [4, Theorem 2.1]. An analytic function $f(z)$ is in the class $\mathcal{S}_{0}(\alpha, \beta, \gamma, p ; A)$ for all proper contraction $A$ with $A \neq \theta$ if and only if

$$
\sum_{k=1}^{\infty}\{k+\beta[p-\gamma+\alpha(k+p)]\} a_{k+p} \leq \beta(p-\gamma+\alpha p)
$$

for $0 \leq \alpha \leq 1,0<\beta \leq 1,0 \leq \gamma<p$, and $p \in \mathbb{N}$.

The result is sharp for the function

$$
f(z)=z^{p}-\frac{\beta(p-\gamma+\alpha p)}{k+\beta[p-\gamma+\alpha(k+p)]} z^{k+p} \quad(k \geq 1) .
$$

THEOREM 1. Let $p>\max \{b-c-1, b-1,-1-c-a\}$ and $a(p+1)>b(a+c)$. If $f(z) \in \mathcal{S}_{0}(\alpha, \beta, \gamma, p ; A)$, then

$$
\begin{aligned}
\left\|I_{0, A}^{a, b, c} f(A)\right\| & \leq \frac{\Gamma(p+1-b+c) \Gamma(p+1)}{\Gamma(p+1-b) \Gamma(a+p+1+c)}\|A\|^{p-b} \\
& +\frac{\beta(p-\gamma+\alpha p) \Gamma(p+1-b+c) \Gamma(p+1)}{\{1+\beta[p-\gamma+\alpha(p+1)]\} \Gamma(p+1-b) \Gamma(a+p+1+c)}\|A\|^{p+1-b}
\end{aligned}
$$

and 


$$
\begin{aligned}
\left\|I_{0, A}^{a, b, c} f(A)\right\| & \geq \frac{\Gamma(p+1-b+c) \Gamma(p+1)}{\Gamma(p+1-b) \Gamma(a+p+1+c)}\|A\|^{p-b} \\
& -\frac{\beta(p-\gamma+\alpha p) \Gamma(p+1-b+c) \Gamma(p+1)}{\{1+\beta[p-\gamma+\alpha(p+1)]\} \Gamma(p+1-b) \Gamma(a+p+1+c)}\|A\|^{p+1-b}
\end{aligned}
$$

for $a>0, b, c \in \mathbb{R}$ and all invertible operator $A$ with $\left(A^{\frac{1}{q}}\right)^{*} A^{\frac{1}{q}}=A^{\frac{1}{q}}\left(A^{\frac{1}{q}}\right)^{*}(q \in \mathbb{N}),\|A\|<1$ and $r_{s p}(A) r_{s p}\left(A^{-1}\right) \leq 1$, where $r_{s p}(A)$ is the radius of spectrum of $A$.

PROOF. Consider the function

$$
\begin{aligned}
F(A) & =\frac{\Gamma(p+1-b) \Gamma(a+p+1+c)}{\Gamma(p+1-b+c) \Gamma(p+1)} A^{b} I_{0, A}^{a, b, c} f(A) \\
& =A^{p}-\sum_{k=1}^{\infty} \frac{\Gamma(k+p+1-b+c) \Gamma(p+1+k) \Gamma(p+1-b) \Gamma(a+p+1+c)}{\Gamma(k+p+1-b) \Gamma(a+k+p+1+c) \Gamma(p+1) \Gamma(p+1-b+c)} a_{k+p} A^{k+p} \\
& =A^{p}-\sum_{k=1}^{\infty} B_{k+p} A^{k+p}
\end{aligned}
$$

where

$$
B_{k+p}=\frac{\Gamma(k+p+1-b+c) \Gamma(p+1+k) \Gamma(p+1-b) \Gamma(a+p+1+c)}{\Gamma(k+p+1-b) \Gamma(a+k+p+1+c) \Gamma(p+1) \Gamma(p+1-b+c)} a_{k+p}
$$

Hence, for convenience, we put

$$
\Phi(k)=\frac{\Gamma(k+p+1-b+c) \Gamma(p+1+k) \Gamma(p+1-b) \Gamma(a+p+1+c)}{\Gamma(k+p+1-b) \Gamma(a+k+p+1+c) \Gamma(p+1) \Gamma(p+1-b+c)} \quad(k \in \mathbb{N}) .
$$

Then, by the constraints of the hypotheses, we note that $\Phi(k)$ is non-increasing for integers $k \geq 1$ and we have $0<\Phi(k)<1$. So $F(z) \in \mathcal{S}_{0}(\alpha, \beta, \gamma, p ; A)$. By Lemma 1, we get

$$
\begin{aligned}
\{1+\beta[p-\gamma+\alpha(p+1)]\} \sum_{k=1}^{\infty} B_{k+p} & \leq \sum_{k=1}^{\infty}\{k+\beta[p-\gamma+\alpha(k+p)]\} B_{k+p} \\
& \leq \sum_{k=1}^{\infty}\{k+\beta[p-\gamma+\alpha(k+p)]\} a_{k+p} \\
& \leq \beta(p-\gamma+\alpha p)
\end{aligned}
$$

which gives

$$
\sum_{k=1}^{\infty} B_{k+p} \leq \frac{\beta(p-\gamma+\alpha p)}{\{1+\beta[p-\gamma+\alpha(p+1)]\}}
$$

Therefore, in a similar way with the proof of [4, Theorem 2.3, p. 305], we obtain

$$
\begin{aligned}
\left\|I_{0, A}^{a, b, c} f(A)\right\| & \geq \frac{\Gamma(p+1-b+c) \Gamma(p+1)}{\Gamma(p+1-b) \Gamma(a+p+1+c)}\left\|A^{-b}\right\|\|A\|^{p} \\
& -\frac{\beta(p-\gamma+\alpha p) \Gamma(p+1-b+c) \Gamma(p+1)}{\{1+\beta[p-\gamma+\alpha(p+1)]\} \Gamma(p+1-b) \Gamma(a+p+1+c)}\|A\|^{p+1}\left\|A^{-b}\right\|
\end{aligned}
$$

and

$$
\begin{aligned}
\left\|I_{0, A}^{a, b, c} f(A)\right\| & \leq \frac{\Gamma(p+1-b+c) \Gamma(p+1)}{\Gamma(p+1-b) \Gamma(a+p+1+c)}\left\|A^{-b}\right\|\|A\|^{p} \\
& +\frac{\beta(p-\gamma+\alpha p) \Gamma(p+1-b+c) \Gamma(p+1)}{\{1+\beta[p-\gamma+\alpha(p+1)]\} \Gamma(p+1-b) \Gamma(a+p+1+c)}\|A\|^{p+1}\left\|A^{-b}\right\| .
\end{aligned}
$$

By equation (7) of $[4, p .307]$, 


$$
\left\|A^{b}\right\|=\|A\|^{b} \quad(b>0) .
$$

Since $A^{*} A=A A^{*},\|A\|=r_{s p}(A)$. So

$$
1=\left\|A A^{-1}\right\| \leq\|A\|\left\|A^{-1}\right\|=r_{s p}(A) r_{s p}\left(A^{-1}\right) \leq 1 .
$$

Thus

$$
\left\|A^{-1}\right\|=\|A\|^{-1}
$$

By (2.9) and (2.10),

$$
\left\|A^{b}\right\|=\|A\|^{b}
$$

for all real $b$. Therefore from (2.7), (2.8) and (2.11) we have the desired estimates.

THEOREM 2. Let $p>\max \{b-c-1, b,-2-c+a\}, c+1<(p-b)(1-a+p+c)$, and $b(2-a+c) \leq(1-a)(1+p)$. If $f(z) \in \mathcal{S}_{0}(\alpha, \beta, \gamma, p ; A)$, then

$$
\begin{aligned}
\left\|D_{0, A}^{a, b, c} f(A)\right\| \leq & \frac{\Gamma(p+1-b+c) \Gamma(p+1)}{\Gamma(p-b) \Gamma(2-a+p+c)}\|A\|^{p-b-1} \\
& +\frac{\beta(p+1)(p-\gamma+\alpha p) \Gamma(p+1-b+c) \Gamma(p+1)}{\{1+\beta[p-\gamma+\alpha(p+1)]\} \Gamma(p-b) \Gamma(2-a+p+c)}\|A\|^{p-b}
\end{aligned}
$$

and

$$
\begin{aligned}
\left\|D_{0, A}^{a, b, c} f(A)\right\| \geq & \frac{\Gamma(p+1-b+c) \Gamma(p+1)}{\Gamma(p-b) \Gamma(2-a+p+c)}\|A\|^{p-b-1} \\
& -\frac{\beta(p+1)(p-\gamma+\alpha p) \Gamma(p+1-b+c) \Gamma(p+1)}{\{1+\beta[p-\gamma+\alpha(p+1)]\} \Gamma(p-b) \Gamma(2-a+p+c)}\|A\|^{p-b}
\end{aligned}
$$

for $0<a<1, b, c \in \mathbb{R}$ and all invertible operator $A$ with $\left(A^{\frac{1}{q}}\right)^{*} A^{\frac{1}{q}}=A^{\frac{1}{q}}\left(A^{\frac{1}{q}}\right)^{*}(q \in \mathbb{N}),\|A\|<1$ and $r_{s p}(A) r_{s p}\left(A^{-1}\right) \leq 1$, where $r_{s p}(A)$ is the radius of spectrum of $A$.

PROOF. Consider the function

$$
\begin{aligned}
G(A) & =\frac{\Gamma(p-b) \Gamma(2-a+p+c)}{\Gamma(p+1-b+c) \Gamma(p+1)} A^{b+1} D_{0, A}^{a, b, c} f(A) \\
& =A^{p}-\sum_{k=1}^{\infty} \frac{\Gamma(k+p+1-b+c) \Gamma(p+1+k) \Gamma(p-b) \Gamma(2-a+p+c)}{\Gamma(k+p-b) \Gamma(2-a+k+p+c) \Gamma(p+1) \Gamma(p+1-b+c)} a_{k+p} A^{k+p} \\
& =A^{p}-\sum_{k=1}^{\infty} C_{k+p} A^{k+p}
\end{aligned}
$$

where

$$
C_{k+p}=\frac{\Gamma(k+p+1-b+c) \Gamma(p+1+k) \Gamma(p-b) \Gamma(2-a+p+c)}{\Gamma(k+p-b) \Gamma(2-a+k+p+c) \Gamma(p+1) \Gamma(p+1-b+c)} a_{k+p}
$$

Hence, for convenience, we put

$$
\Psi(k)=\frac{\Gamma(k+p+1-b+c) \Gamma(p+k) \Gamma(p-b) \Gamma(2-a+p+c)}{\Gamma(k+p-b) \Gamma(2-a+k+p+c) \Gamma(p+1) \Gamma(p+1-b+c)} \quad(k \in \mathbb{N}) .
$$

Then, by the constraints of the hypotheses, we note that $\Psi(k)$ is non-increasing for integers $k \geq 1$ and we have $0<\Psi(k)<1$, i.e.,

$$
0<\frac{\Gamma(k+p+1-b+c) \Gamma(p+1+k) \Gamma(p-b) \Gamma(2-a+p+c)}{\Gamma(k+p-b) \Gamma(2-a+k+p+c) \Gamma(p+1) \Gamma(p+1-b+c)}<k+p
$$

Also, by the relation

$$
\frac{k+p}{p+1}\{1+\beta[p-\gamma+\alpha(p+1)]\} \leq k+\beta[p-\gamma+\alpha(p+k)] \quad(k \geq 1),
$$

we get 


$$
\begin{aligned}
\sum_{k=1}^{\infty} \frac{k+p}{p+1}\{1+\beta[p-\gamma+\alpha(p+1)]\} \Psi(k) a_{k+p} & \leq \sum_{k=1}^{\infty}\{k+\beta[p-\gamma+\alpha(k+p)]\} \Psi(k) a_{k+p} \\
& \leq \beta(p-\gamma+\alpha p),
\end{aligned}
$$

that is,

$$
\sum_{k=1}^{\infty}(k+p) \Psi(k) a_{k+p} \leq \frac{\beta(p+1)(p-\gamma+\alpha p)}{\{1+\beta[p-\gamma+\alpha(p+1)]\}} .
$$

Therefore, in the same way with the proof of Theorem 1, we obtain

$$
\begin{aligned}
\left\|D_{0 . A}^{a, b, c} f(A)\right\| \leq & \frac{\Gamma(p+1-b+c) \Gamma(p+1)}{\Gamma(p-b) \Gamma(2-a+p+c)}\|A\|^{p-b-1} \\
& +\frac{\Gamma(p+1-b+c) \Gamma(p+1)}{\Gamma(p-b) \Gamma(2-a+p+c)}\|A\|^{p-b} \sum_{k=1}^{\infty}(k+p) \Psi(k) a_{k+p} \\
\leq & \frac{\Gamma(p+1-b+c) \Gamma(p+1)}{\Gamma(p-b) \Gamma(2-a+p+c)}\|A\|^{p-b-1} \\
& +\frac{\beta(p+1)(p-\gamma+\alpha p) \Gamma(p+1-b+c) \Gamma(p+1)}{\{1+\beta[p-\gamma+\alpha(p+1)]\} \Gamma(p-b) \Gamma(2-a+p+c)}\|A\|^{p-b}
\end{aligned}
$$

and

$$
\begin{aligned}
\left\|D_{0, A}^{a, b, c} f(A)\right\| & \geq \frac{\Gamma(p+1-b+c) \Gamma(p+1)}{\Gamma(p-b) \Gamma(2-a+p+c)}\|A\|^{p-b-1} \\
& -\frac{\beta(p+1)(p-\gamma+\alpha p) \Gamma(p+1-b+c) \Gamma(p+1)}{\{1+\beta[p-\gamma+\alpha(p+1)]\} \Gamma(p-b) \Gamma(2-a+p+c)}\|A\|^{p-b}
\end{aligned}
$$

REMARK. (i) By the proof of Theorem 1, if we put

$$
F(z) \equiv J_{0, z, p}^{a, b, c} f(z):=\frac{\Gamma(p+1-b) \Gamma(a+p+1+c)}{\Gamma(p+1-b+c) \Gamma(p+1)} z^{b} I_{0, z}^{a, b, c} f(z),
$$

then we know that $J_{0, z, p}^{a, b, c}$ is a fractional linear operator from $\mathcal{S}_{0}(\alpha, \beta, \gamma, p)$ to itself.

(ii) From (1.17) it is easy to see that Theorem 1 and Theorem 2 are generalizations of $[4$, Theorem 3.1 and Theorem 3.2].

ACKNOWLEDGEMENT. The authors were partially supported by KOSEF (94-0701-02-01-3) and TGRC-KOSEF, and by the Basic Science Research Institute Program, Ministry of Education, 1994 (BSRI-96-1401).

\section{REFERENCES}

[1] LEE, S.H., KIM, Y.C and CHO, N.E., A subclass of analytic functions with negative coefficients, Math. Japonica 34 (1989), 597-605.

[2] DUNFORD, N. and SCHWARTZ, J.T., Linear Operators, Part I, General Theory, Interscience, New York, 1958.

[3] FAN, K., Julia's lemma for operators, Math. Ann. 239 (1979), 241-245.

[4] XIAOPEI, Y., A subclass of analytic p-valent functions for operator on Hilbert space, Math. Japonica 40 (1994), 303-308.

[5] SRIVASTAVA, H.M., SAIGO, M. and OWA, S.A., A class of distortion theorems involving certain operators of fractional calculus, J. Math. Anal. Appl. 131 (1988), 412-420.

[6] SRIVASTAVA H.M. and OWA, S. (Editors), Univalent Functions, Fractional Calculus, and Their Applications, Halstead Press (Ellis Horwood Limited, Chichester), John Wiley and Sons, New York, Chichester, Brisbane and Toronto, 1989.

[7] OWA, S., SAIGO, M. and SRIVASTAVA, H.M., Some characterization theorems for starlike and convex functions involving a certain fractional integral operator, J. Math. Anal. Appl. 140 (1989), 419-426.

[8] KIM, Y.C. PARK, Y.S. and SRIVASTAVA H.M., A subclass of inclusion theorems associated with some fractional integral operators, Proc. Japan Acad Ser. A Math. Sci. 67 (1991), 313-318.

[9] SOHI, N.S., Distortion theorems involving certain operators of fractional calculus on a class of pvalent functions, in Fractional Calculus and its Applications (K. Nishimoto, Ed.); Some characterization and distortion theorems involving fractional calculus, generalized hypergeometric functions, College of Engineering (Nihon University), Koriyama, 1990, pp. 245-252. 


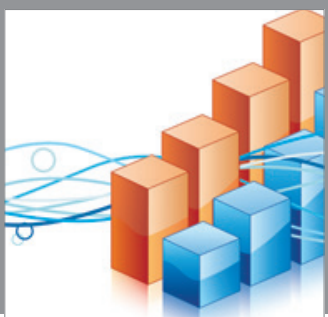

Advances in

Operations Research

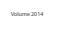

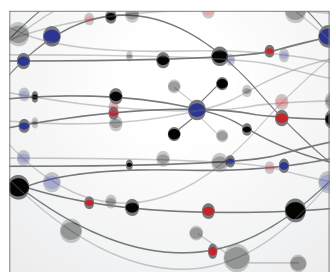

\section{The Scientific} World Journal
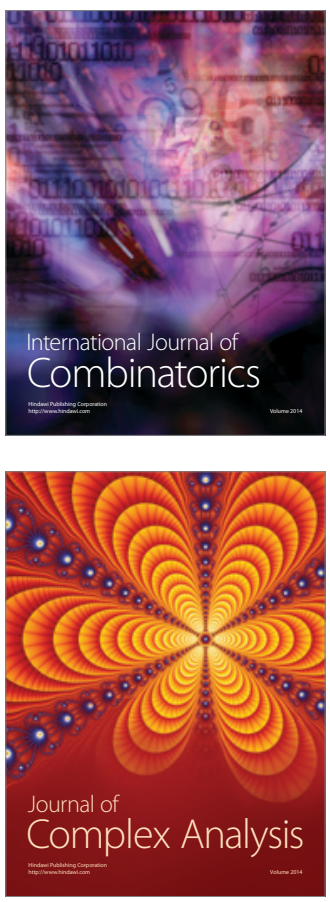

International Journal of

Mathematics and

Mathematical

Sciences
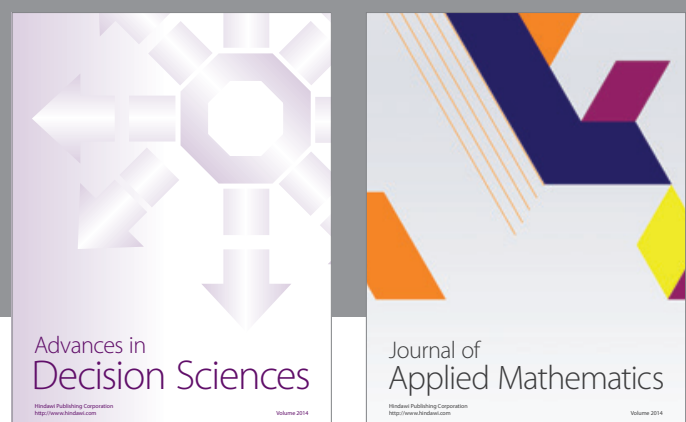

Journal of

Applied Mathematics
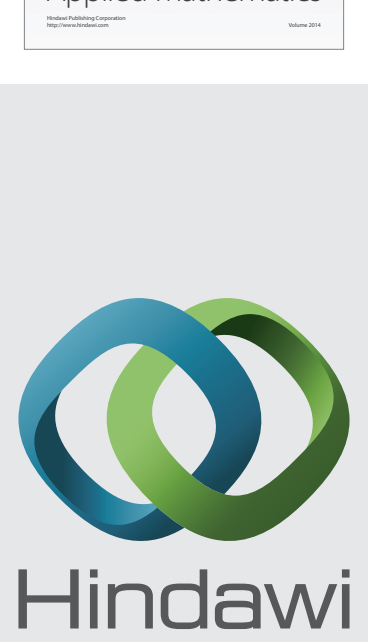

Submit your manuscripts at http://www.hindawi.com
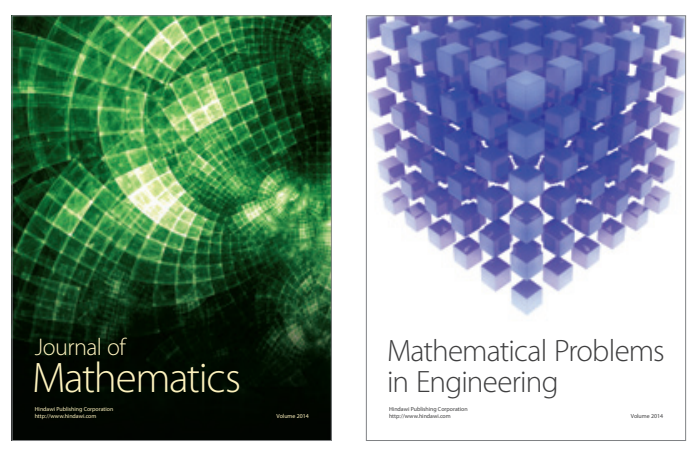

Mathematical Problems in Engineering
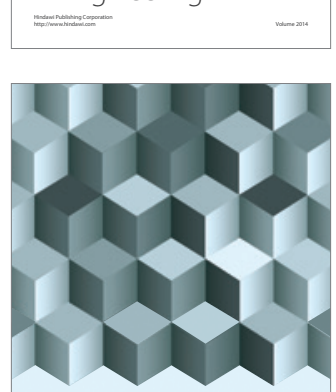

Journal of

Function Spaces
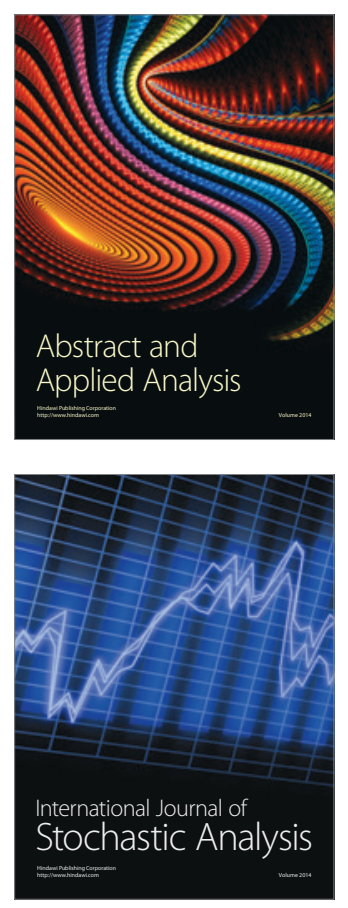

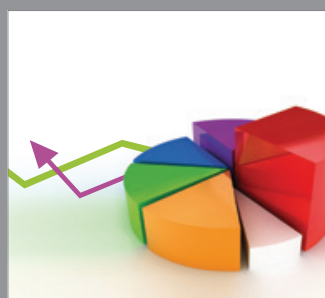

ournal of

Probability and Statistics

Promensencen
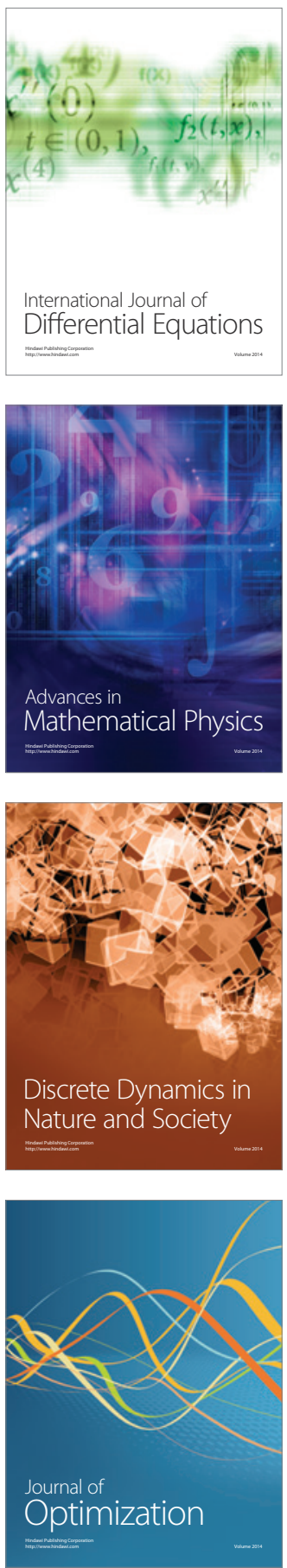